\title{
Discharge and velocity variation of flows in open channels partially covered with different layered vegetation
}

\author{
Xiaonan Tang*, Yutong Guan, Hamidreza Rahimi, Prateek Singh, and Yujia Zhang \\ Department of Civil Engineering, Xi' an Jiaotong-Liverpool University, 111 Ren'ai Road, SIP Suzhou, 215123, China
}

\begin{abstract}
The role of vegetation in the natural environment has drawn great interest recently. The vegetation can change the velocity distribution due to its additional resistance on the flow, consequently affecting the nutrient and pollutant transport, and the habitats. Due to the complexity of flow and vegetation interaction, many previous researchers have studied the flow structure of channels with uniform vegetation. Few studies have been done on the flow of open channels partially covered vegetation of different heights on one side of the channel, which commonly exists in natural rivers. Through novel experiments for such a vegetated flow, this paper shows the influence of different layered vegetation on the velocity profile and discharge, which indicates that the velocity in the vegetation zone is significantly smaller than that in the free-flow zone and that the velocity profiles in the short and tall vegetation zones are very different. The flow through the free-flow zone is dominant (about $75 \%$ ) despite its half width, and its discharge percentage slightly decreases as increasing flow depth.
\end{abstract}

\section{Introduction}

The significance of aquatic vegetation in the river ecosystem has drawn much attention. The vegetation can improve water quality, protect the embankment, and provide a suitable environment for biodiversity and habitat creation [1-5].

Many researchers have undertaken studies on vegetated flow in the last few decades, mainly focusing on single-layered vegetation. These studies include the prediction of flow resistance [6-7] and vertical velocity distribution [8-12], the modelling of the lateral variation of depth averaged velocity [13-15] in an open channel with single-layered vegetation, together with the modelling of the longitudinal profiles of velocity [16]. Meanwhile, spatial development of coherent motion was studied in an open-channel of limited length vegetation [17]. White and Nepf [18] experimentally studied vertical velocity distributions, shear stresses, and boundary layer widths in a wide channel partially covered single-layer vegetation of wooden cylinders in emergent conditions, indicating that a distinct layer flow structure exists with a great lateral velocity gradient over the interface between the vegetation and free flow zones.

In many natural rivers, the vegetation of different heights, such as grasses, shrubs and trees, commonly exists in certain zones along the river [19]. Under certain flow conditions, tall vegetation is not submerged, but some short vegetation is submerged. Thus, the flow structure between different flow zones becomes complex because of the momentum exchange between the flows through different layered vegetation. For understanding the flow structure of channels with mixing-layered vegetation, some experimental studies were done in an open channel fully covered with a coexistence of tall and short vegetation (e.g. [20-23]). Most recently, Tang et al. [24-26] conducted some experimental studies on the flow characteristics of a channel with two-layered vegetation that covered part of the channel bed. These results show that the velocity profiles are significantly different from those in singlelayered vegetation, and a large lateral velocity variation exists across the vegetation interface. However, it is unknown how the velocity and discharge change when a channel bed is partially covered with vegetation of different heights, which commonly exists in natural rivers or ecologically designed channels. This paper is to understand the velocity variation of flows in such a vegetated channel.

In the present study, a novel vegetated flow experiment was undertaken in a tilting rectangular flume. The flume bed is half occupied by a row of tall vegetation alongside another row of short vegetation. The velocities at different positions were measured to reveal the flow structure in the zones of non-vegetation and vegetation. This paper mainly focuses on the variation of velocity profiles and discharge percentage across different zones when the vegetation is in emergent, partially and fully submerged conditions.

\section{Methods}

\subsection{Experimental setting}

The experiment was undertaken in a straight tilting flume at the fluid mechanics' lab of Xi'an Jiaotong-

\footnotetext{
* Corresponding author: xiao.tang@xjtlu.edu.cn
} 
Liverpool University (XJTLU). The $20 \mathrm{~m}$ long rectangular flume is $0.4 \mathrm{~m}$ wide and was set with a bed slope of 0.003 [24]. The uniform flow in the test section can be achieved through an adjustable tailgate at the outlet of the flume, where a few small mesh boards are placed at the flume's entrance to reduce the disturbance of inflow.

In our experiments, rigid vegetation was modelled by PVC dowels of two heights: $20 \mathrm{~cm}$ and $10 \mathrm{~cm}$, which respectively represent the tall and short plant. All dowels have a diameter of $6.35 \mathrm{~mm}$. Pre-perforated PVC sheets for holding the dowels were put on the bed of the flume.

The $7.2 \mathrm{~m}$ long vegetation area on the left side (Fig. 1) started $9 \mathrm{~m}$ downstream from the entrance of the flume [25]. Figure 1 shows different measuring positions in a cross-section. The plus signs indicate the measuring location, the red star circles denote short vegetation, and the black circles denote tall vegetation. A mini propeller velocimetry was used to measure velocity for each measuring point at least twice, and the sampling time was 20 seconds. The measuring locations are shown in Table 1.

Table 1. The summary of measuring positions

\begin{tabular}{llll}
\hline \multicolumn{2}{l}{ Vegetated Zone } & \multicolumn{2}{l}{ Free-Flow Zone } \\
\hline Measuring & Distance to & Measuring & Distance to \\
position & Wall A (mm) & position & Wall A (mm) \\
W1 & 12.7 & W13 & 200.0 \\
W2 & 25.4 & W14 & 215.9 \\
W3 & 41.3 & W15 & 247.6 \\
W4 & 57.1 & W16 & 279.4 \\
W5 & 73.0 & W17 & 295.3 \\
W6 & 88.9 & W18 & 311.1 \\
W7 & 104.8 & W19 & 327.0 \\
W8 & 120.6 & W20 & 342.9 \\
W9 & 136.5 & W21 & 358.8 \\
W10 & 152.4 & W22 & 374.6 \\
W11 & 168.3 & W23 & 387.3 \\
W12 & 184.1 & & \\
W25 & 73.0 & & \\
W26 & 104.8 & & \\
W27 & 136.5 & & \\
\hline
\end{tabular}

\subsection{Experimental flow conditions}

Three flow depths of 9,18 , and $22 \mathrm{~cm}$ were conducted in our experiments. They respectively represent the following three flow conditions: (1) two types of dowels are emergent, (2) the tall dowels are emergent, but the short dowels are submerged, and (3) all dowels are completely submerged.

For understanding the subsequent figures in the Results section, the velocity is normalised by the shear velocity $\mathrm{u} *$, given by $\sqrt{\mathrm{g} H S}$, where $\mathrm{g}$ is the gravitational acceleration, $H$ is the depth of flow, and $S$ is the channel bed slope. The vertical distance (z) above the channel bottom is also normalized by the height of short vegetation $\left(h_{s}\right)$.

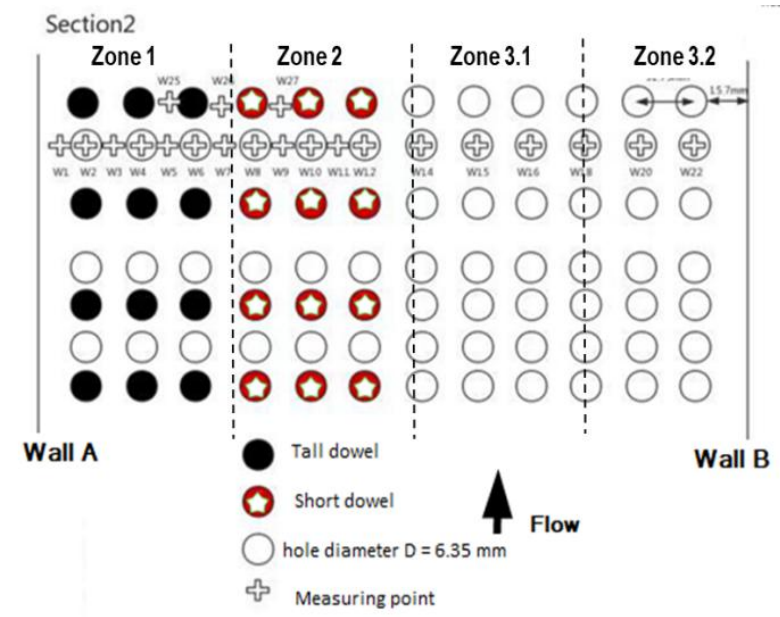

Fig. 1. Dowel arrangement and measuring points

\section{Results and discussion}

\subsection{Longitudinal velocity}

According to the submergence state of vegetation under three flow depths of 9,18 and $22 \mathrm{~cm}$, the vertical velocity distributions are categorized into emergent, partially-submerged, and fully-emergent conditions. Figures 2-5 show the experimental results of averaged velocity profiles. In the figures, WM1-6 denotes the averaged value of velocity in the tall vegetation (Zone 1), WM7-12 denotes the averaged velocity in the short vegetation (Zone 2), W13-17 denotes the averaged velocity in the inner zone of the free flow (Zone 3.1), and $\mathrm{W} 17-23$ denotes the averaged velocity in the outer zone of the free-flow (Zone 3.2). W12-23 presents the averaged velocity in the entire free flow zone.

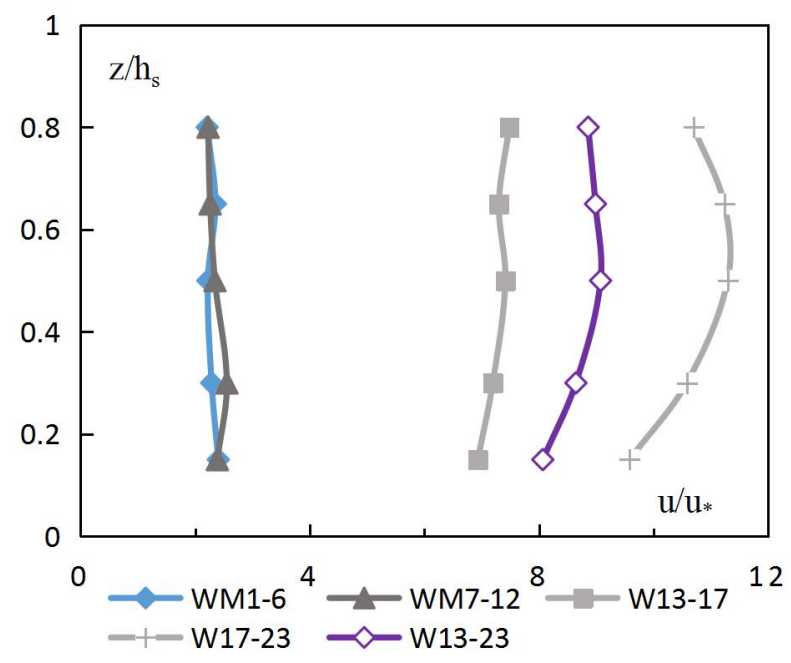

Fig. 2. Velocity profiles for flow depth of $9 \mathrm{~cm}$ in emergent conditions.

For the emergent condition (Fig. 2), the vertical variation of velocity is small in the vegetation zone (Zone 1 and 2), which implies that the vertical momentum exchange of flow is very weak. However, the velocity appears to increase vertically in the free zone (Zone 3), and the vertical increase in velocity is more prominent in Zone 3.1 than in Zone 3.2. In other 
words, the vertical variation of velocity in the far end zone from the vegetation is more significant than that in the zone near the vegetation. Furthermore, the overall velocity profile in Zone 3.2 is much larger than that in Zone 3.1, which is significantly larger than that in the vegetation zone. This result shows that the vegetation decreases the flow velocity through the vegetation, which is not surprising because of the additional friction of vegetation to the flow, as observed by other researchers $[6,21]$. Furthermore, the vegetation influences the free zone's flow, revealing the smaller velocity in the zone close to the vegetation than that in the zone far from the vegetation.

For the partially-submerged flow condition (Fig. 3), i.e., the tall vegetation in Zone 1 is emergent while the short vegetation in Zone 2 is submerged, the velocity in Zone 1 is almost constant over the depth, where the drag force dominates the flow compared with the bed shear stress and turbulent shear stress. However, the velocity in Zone 2 (in submerged conditions) remains constant near the bed. It then increases fast towards the top edge of short vegetation, thereby gradually increases until the water surface.

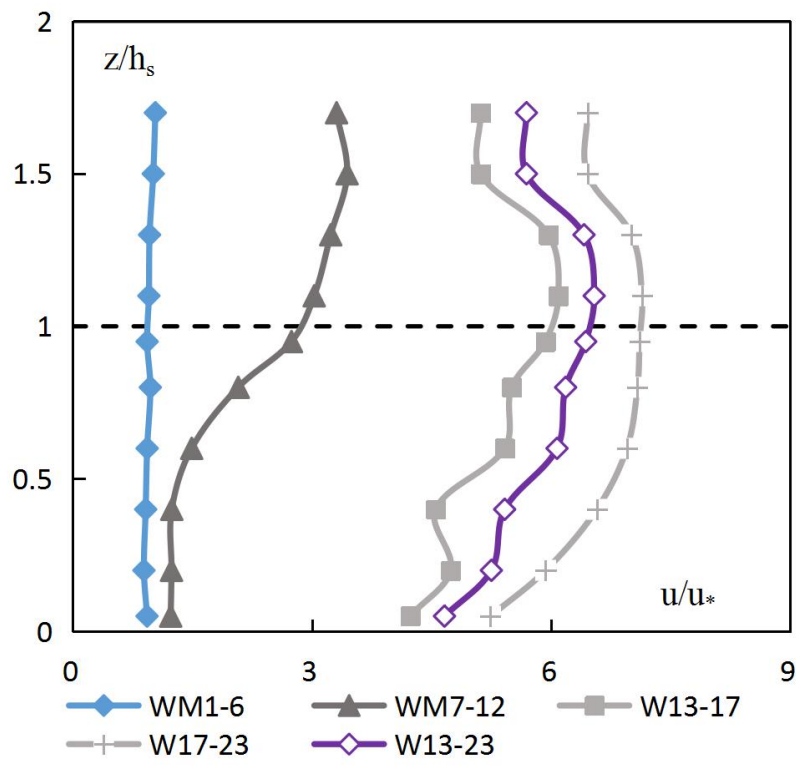

Fig. 3. Velocity profiles for flow depth of $18 \mathrm{~cm}$ in partiallysubmerged conditions.

Like the emergent flow condition, the velocity in the free-flow zone is affected by the vegetation, and a maximum velocity appears somewhere $\left(\mathrm{z} / \mathrm{h}_{\mathrm{s}}=1.3\right)$ above the short vegetation.

For the fully-submerged flow condition (Fig. 4), i.e., both the tall and short vegetation are in submerged conditions. Because of the different submergence ratios in Zone 1 (tall vegetation) and Zone 2 (short vegetation), the velocity increases rapidly at a certain distance from the bed. The velocity starting change is about $\mathrm{z} / \mathrm{h}_{\mathrm{s}}=1.6\left(\right.$ or $\mathrm{z} / \mathrm{h}_{\mathrm{t}}=0.8$, where $\mathrm{h}_{\mathrm{t}}$ is the height of tall vegetation) in Zone 1 , whereas it occurs at $\mathrm{z} / \mathrm{h}_{\mathrm{s}}=$ 0.6 in Zone 2. Although the velocity profile in the freeflow zone (Zone 3) shows a maximum value at $\mathrm{z} / \mathrm{h}_{\mathrm{s}}$ $=1.1$, it appears that the lateral influence of vegetation on the velocity in zone 3 becomes smaller.

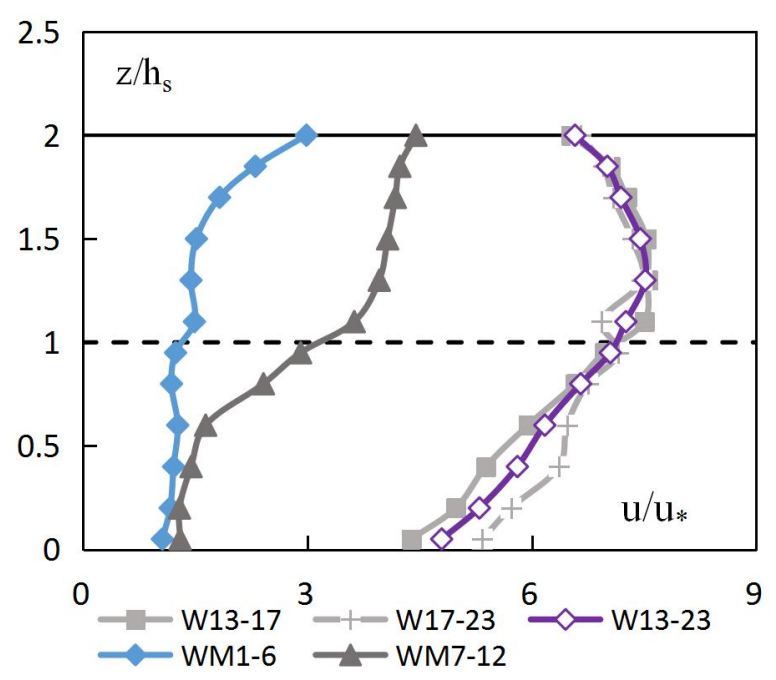

Fig. 4. Velocity profiles for flow depth of $22 \mathrm{~cm}$ in fullysubmerged conditions.

Compared with the three conditions (Figs. 2-4), with increasing flow depth, the velocity in the tall vegetation zone (zone 1) is almost constant over the depth in emergent conditions. Still, it starts to increase from a certain distance below the vegetation top when the vegetation is submerged. The velocity profile in Zone 2 shows a distinct transition from the vegetated layer to the water surface layer, in which the velocity gradually increases, but the change of velocity is significantly over the edge of short vegetation. This result indicates that large momentum exchanges exist between the two layers. The velocity profiles in the free-flow zone (Zone 3 ) are primarily affected by the vegetation. The smaller the flow depth, the more significant the impact of vegetation becomes.

Besides, as the depth increases, the vegetation mainly affects the velocity profiles in the zone above the short vegetation, showing a large velocity for a higher depth (Fig. 5).

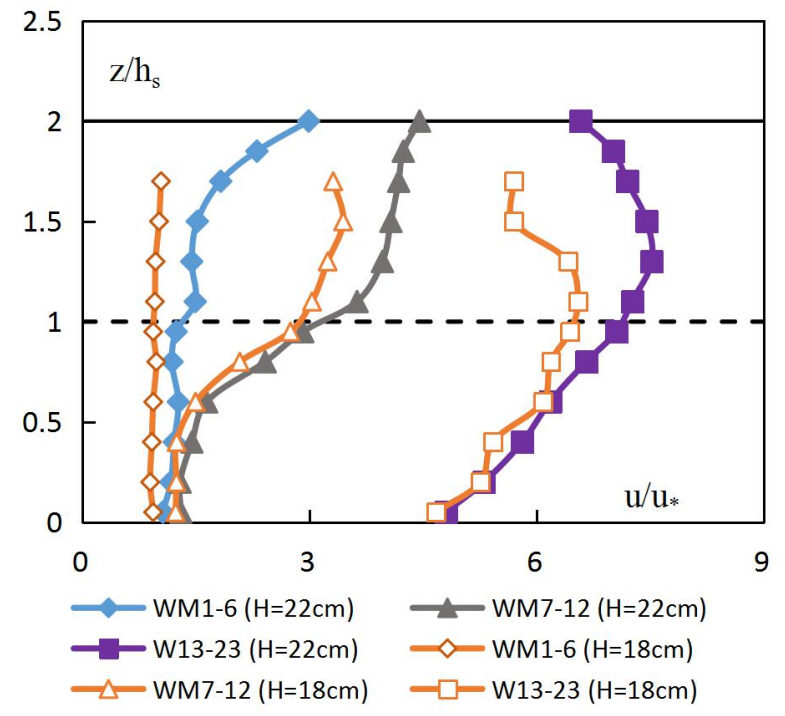

Fig. 5. Comparison of velocity profiles for flow depths of 18 and $22 \mathrm{~cm}$

3.2 Discharge percentage in each zone 
The discharge in each zone can be computed from the lateral distribution of depth averaged velocity in a channel cross section. Figure 6 presents the percentage of discharge in each zone for the three flow depths. The data in Figure 6 reveal that the discharge in the free-flow zone (Zone 3) is dominant, almost takes 73$79 \%$ of the total discharge, whereas the discharge through the vegetated zone only takes approximately $30-20 \%$ of the total discharge, although the vegetated zone has the same width as the non-vegetated zone. This result implies that the vegetation significantly decreases the flow velocity through the vegetation, resulting in a much smaller portion of flow within the vegetation, which will help the deposition of nutrients and sediment.

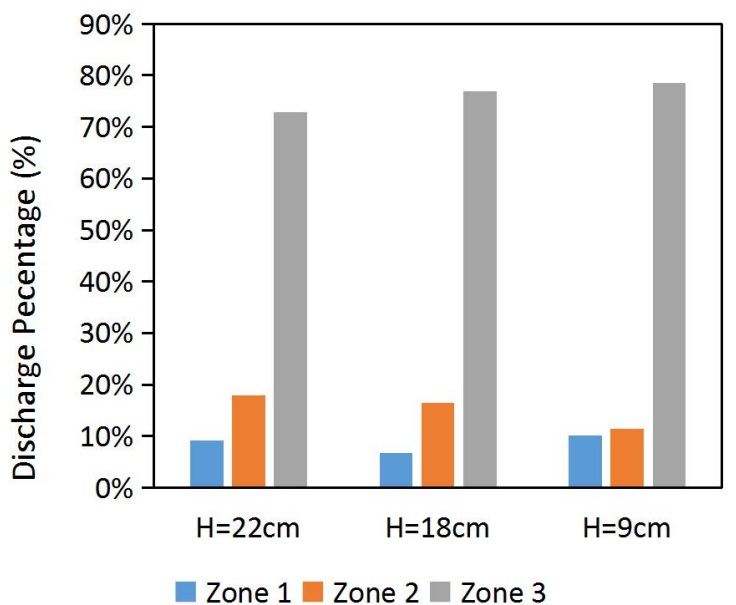

Fig. 6. Discharge percentage in each zone of three flow depths: $9 \mathrm{~cm}, 18 \mathrm{~cm}, 22 \mathrm{~cm}$

With increased water depth from $9 \mathrm{~cm}$ to $22 \mathrm{~cm}$, the discharge in the free flow zone (zone 3) decreases slightly from $79 \%$ to $73 \%$. In contrast, the discharge in zone 2 (i.e. the short vegetation zone) increases from $11.5 \%$ to $18 \%$, but the discharge in zone 1 (i.e., the tall vegetation zone) varies without a clear tendency. The relative submergence of vegetation may cause an increase in discharge percentage in zone 2 . When the flow depth changes from $9 \mathrm{~cm}$ to $14 \mathrm{~cm}$, the short vegetation in zone 2 changes from emergent to fully submerged, which imposes less resistance due to the vegetation drag, consequently having a relatively large velocity through the vegetation. When the flow depth further increases from $18 \mathrm{~cm}$ to $22 \mathrm{~cm}$, the short vegetation in zone 2 has higher submergence, consequently, less resistance from the drag force of vegetation, further increasing the velocity of flow through the short vegetation, and has a relatively large discharge.

For zone 1 (i.e., tall vegetation), when the vegetation in emergent conditions $(\mathrm{H}=9 \mathrm{~cm}$ and $18 \mathrm{~cm})$, the flow resistance due to the drag force increases as the flow increase, the discharge decreases. However, when the flow depth increases from $18 \mathrm{~cm}$ to $22 \mathrm{~cm}$, the submergence state of the vegetation in zone 1 has substantial change from emergent to fully submerged, the additional flow resistance from the vegetation becomes smaller, leading to an increase of discharge in the higher flow $(\mathrm{H}=22 \mathrm{~cm})$.

\section{Conclusions}

The observed velocity results of flow in an open channel partially covered with two heights of vegetation and under three different flow conditions show:

- The vegetation retards flow through the vegetation. Under emergent conditions, the velocity of flow in the vegetation has a small variation over the depth, where the drag force takes the dominant role in the flow.

- In the partially-submerged condition, the velocity profile in the zone of short vegetation (zone 2) shows a rapid increase over the transition layer from the lower vegetated layer to the upper surface layer, which is completely different from the velocity profile in the zone of tall vegetation (zone $1)$. The vegetation also affects the velocity profile in the free-flow zone (zone 3), where a maximum velocity occurs above the short vegetation, approximately at $\mathrm{z} / \mathrm{h}_{\mathrm{s}}=1.3$.

- For the fully-submerged condition, the velocity profile in zone 2 is similar to that in the partiallysubmerged condition, but the velocity gradient in the transition layer increases when the submergence ratio $\left(H / h_{s}\right)$ increases. The velocity profile in the zone of free flow (zone 3) has a maximum value occurring well below the water surface.

- It appears that the increase of flow depth will affect the velocity profiles above the short vegetation (Fig. 5).

- The free-flow zone will carry most flow (73-79\%), although it has only half the channel's width. As the flow depth increases, the discharge percentage in the free-flow zone (zone 3) will decrease slightly, but the discharge percentage in zone 2 increases gradually, whereas it varies in zone 1 .

\section{Acknowledgments}

The first author would acknowledge the support by XJTLU via the Research Funds (RDF-16-02-02, KSF-E-17, KSF-A19) and by the National Natural Science Foundation of China (11772270).

\section{References}

[1] J. Curran, W. Hession, Vegetative impacts on hydraulics and sediment processes across the fluvial system, Journal of Hydrology, 505, 364376 (2013)

[2] J. Zhang, W. Huai, H. Shi, W. Wang, Estimation of the longitudinal dispersion coefficient using a two- zone model in a channel partially covered with artificial emergent vegetation, Environmental Fluid Mechanics, (2020), DOI: 10.1007/s10652-020-09766-2

[3] H. Nepf, E. Vivoni, Flow structure in depthlimited vegetated flow, Journal of Geophysical 
Research: Oceans, 105 (C12): 28547-57 (2000)

[4] J. Gu, Y. Shan, C. Liu, X. Liu, Feedbacks of flow and bed morphology from a submerged dense vegetation patch without upstream sediment supply, Environmental Fluid Mechanics, 19 (2), 475-493 (2019)

[5] E.M. Follett, H.M. Nepf, Sediment patterns near a model patch of reedy emergent vegetation, Geomorphology, 179, 141-151 (2012)

[6] B. M. Stone, H. T. Shen, Hydraulic resistance of flow in channels with cylindrical roughness, Journal of Hydraulic Engineering, 128 (5), 500506 (2002)

[7] F. Yang, W. Huai, Y. Zheng, New dynamic twolayer model for predicting depth-averaged velocity in open-channel flows with rigid submerged canopies of different densities, Advances in Water Resources, 138. 103553 (2020)

[8] X. Tang, S. Ali, Evaluation of methods for predicting velocity profiles in open channel flows with submerged rigid vegetation, in Proceedings of the 35th IAHR World Congress, 4, B1, Sept. 813, 2013, Chengdu, China (2013)

[9] X. Tang, A mixing-length-scale-based analytical model for predicting velocity profiles of openchannel flows with submerged rigid vegetation, Water and Environment Journal, 33 (4): 610-619 (2019)

[10] X. Tang, Evaluating two-layer models for velocity profiles in open-channels with submerged vegetation, Journal of Geoscience and Environment Protection, 7 (1): 68-80 (2019)

[11] W. Huai, Y. Zeng, Z. Xu, Z. Yang, Three-layer model for vertical velocity distribution in open channel flow with submerged rigid vegetation, Advances in Water Resources, 32 (4), 487-492 (2009)

[12] X. Tang, An improved analytical model for vertical velocity distribution of vegetated channel flows, Journal of Geoscience and Environment Protection, 7 (4): 42-60 (2019)

[13] X. Tang, D. W. Knight, Lateral distributions of streamwise velocity in compound channels with partially vegetated floodplains, Science in China Series E: Technological Sciences, 52 (11): 33573362 (2009)

[14] X. Tang, M. Sterling, D. Knight, A general analytical model for lateral velocity distributions in vegetated channels, in River Flow 2010: 469476, (Karlsruhe, 2010)

[15] X. Tang, D. W. Knight, M. Sterling, Analytical model of streamwise velocity in vegetated channels, Proceedings of the Institute of Civil Engineers: Engineering and Computational Mechanics, 164 (2), 91-102 (2011)

[16] C. Yan, Y. Shan, W. Sun, C. Liu, X. Liu, Modeling the longitudinal profiles of streamwise velocity in an open channel with a model patch of vegetation, Environmental Fluid Mechanics, 20:
1441-1462 (2020)

[17] T. Okamoto, I. Nezu, Spatial evolution of coherent motions in finite-length vegetation patch flow, Environmental Fluid Mechanics, 13, 417434 (2013)

[18] B. L. White, H. M. Nepf, A vortex-based model of velocity and shear stress in a partially vegetated shallow channel, Water Resources Research, 44 (1): W01412 (2008)

[19] V. Chembolu, R. Kakati, S. Subashisa Dutta, A laboratory study of flow characteristics in natural heterogeneous vegetation patches under submerged conditions, Advances in Water Resources, 133: 103418 (2019)

[20] D. Liu, P. Diplas, C. C. Hodges, J. D. Fairbanks, Hydrodynamics of flow through double layer rigid vegetation, Geomorphology, 116 (3-4), 286296 (2010)

[21] H. Rahimi, X. Tang, P. Singh, M. Li, S. Alaghmand, Open channel flow within and above a layered vegetation: Experiments and first-order closure modelling, Advances in Water Resources, 137 (3): 103527 (2020)

[22] P. Singh, H. Rahimi, X. Tang, Parameterization of the modeling variables in velocity analytical solutions of open-channel flows with doublelayered vegetation, Environmental Fluid Mechanics, 19 (3): 765-784 (2019)

[23] H. Rahimi, X. Tang, P. Singh, Experimental and numerical study on impact of double layer vegetation in open channel flows, Journal of Hydrologic Engineering, 25 (2): 04019064 (2020)

[24] X. Tang, Y. Guan, Y. Zhang, W. Zhang, T. Liu, X. Yi, Effect of vegetation on the Flow of a partiallyvegetated channel. IOP Conf. Series: Earth and Environmental Science, 668, 012050, IOP Publishing (2021), DOI:10.1088/1755$1315 / 668 / 1 / 012050$

[25] X. Tang, H. Rahimi, Y. Guan, Y. Wang, Hydraulic characteristics of open-channel flow with partially-placed double layer vegetation, Environmental Fluid Mechanics, 2021, DOI: 10.1007/s10652-020-09775-1.

[26] X. Tang, H. Rahimi, P. Singh, Z. Wei, Y. Wang, Y. Zhao, Experimental study of open-channel flow with partial double-layered vegetation, in Proceeding of E3S Web of Conferences (EDP Sciences, 2019) 